\title{
Fabrication of Aligned Polylactic Acid Porous Fibers by Electrospinning
}

\author{
Yanhua Song ${ }^{1, ~ a, ~ L a n ~} \mathrm{Xu}^{2, \mathrm{~b}}$ \\ ${ }^{1}$ National Engineering Laboratory for Modern Silk, College of Textile and \\ Clothing Engineering, Soochow University, Suzhou, 215123, China \\ ${ }^{2}$ National Engineering Laboratory for Modern Silk, College of Textile and \\ Clothing Engineering, Soochow University, Suzhou, 215123, China \\ aemail: syanhua2015@163.com, bemail: lanxu@suda.edu.cn (corresponding author)
}

\begin{abstract}
Keywords: Electrospinning; Aligned fibers; Nanoporous fibers; Polylactic acid
Abstract. The aligned polylactic acid (PLA) porous fibers have a feature of high specific surface area, special structure and excellent biological properties, which are appropriate as the material of tissue engineering. Simultaneously, the aligned PLA porous fibers are beneficial to the orientation of tissue cells, so it is expected to become a good biological scaffold material. The solvent was composed by of chloroform $(\mathrm{CF})$ and N,N-dimethylformamide (DMF) of which the ratio was 9:1, and the concentration of PLA was eight percent. The obtained fibers were observed under the scanning electron microscope and indicated that they were porous and paralleled.
\end{abstract}

\section{Introduction}

Electrospinning is an effective technique that produces fibers [1]. Electrospun fibers can be used in many fields, for instance, fibers films can be utilized directly as a sensor to improve the sensitivity, electrospun fibers can be used as electrode material, also scaffold of different cells [2,3], however, there are still some urgent issues to tackle because of the limit. One of the reasons that restricts the application and development of electrospinning is the obtained fibers by traditional spinning device is random. However, aligned fibers give the fibers distinct properties of optics, electricity and magnetism, which extend the application range of electrospinning. At present, there are many spinning methods fabricating ordered fibers, such as method of additional magnetic field [4], method of additional ring [5], drum-type spindle method, method of parallel [6], turntable method, supplementary electric field method et al, every method has both its advantages and disadvantages. Li [7] et al prepared paralleled polyvinylpyrrolidone fibers by two paralleled received substance silicon wafer.

The growth direction of cells is flexible and optical, so it is vital for the oriented differentiation of neuron, muscle cell and vascular endothelial cell (VEC) et al. that the ground substance is directional of mechanical properties and chemical structure. Zhong [8] repopulated rabbit conjunctival fibroblasts on well-aligned collagenous fiber and he found a higher cellproliferation rates because of the high-aligned collagenous fiber substance. Human coronary artery smooth muscle cells (SMCs) was cultured on aligned poly(L-lactid-co-å-caprolactone) [P(LLA-CL)] copolymer nanofibrous scaffold by $\mathrm{Xu}$ [9] et al. As a result, compared with on the plane polymer films, SMCs on the aligned nanofibrous scaffold had a remarkable increase of the adhesion and proliferation rate. Moreover, cell infiltration capacity can be effectively improved by preparing composite aligned porous scaffold. Tissue ingrowth needs a highly porous micro-structure with interconnected pores and large surface area [10].

Resent years have witnessed the widely research of porous fibers because of its ultrahigh surface area and high porosity [11]. Polylactic acid (PLA) is an extensively used biomaterial due to its good biocompatibility and mechanical properties [12]. Also, it is degradable and "can be synthesized from renewable resources through bioconversionand polymerization "[13]. So, the porous structure of PLA can be drug-loading.

In this paper, a modified parallel electrode method (MPEM) by placing a positively charged ring between the needle and the parallel electrode collector was introduced for the preparation of well-aligned porous PLA fibers. 


\section{Experimental Section}

Materials. PLA was used as the solvend for preparing porous material, which molecular weight was $100,000 \mathrm{~g} / \mathrm{mol}$. We chose chloroform (CF) and N,N-dimethylformamide(DMF) as solvent system, and they were purchased respectively from Qiang Sheng Functional Chemical Co.,Ltd. Jiangsu province and Ling Feng Chemical Reagent Co.,Ltd. ,Shanghai province. The solution system was prepared at a concentration of $8 \mathrm{wt} \%$ with the proportion of 9:1 of $\mathrm{CF}$ and DMF. The solution was magnetically stirred for 4 hours at room temperature.

Instrument. The electrospinning device included a flux pump, an injector, a copper ring, two straight electrodes and two high voltage DC power supply ( 0-30 kV, DW-P303-1ACF0, Tianjin DongWen high-voltage power generator Co., LTD ). The point of the needle was connected with the positive pole of the high voltage dc power supply 1, and the copper ring was connected with the positive pole of high voltage dc power supply 2 , and the parallel electrode the negative pole of the high voltage dc power supply 1, as is showed in Fig.1.

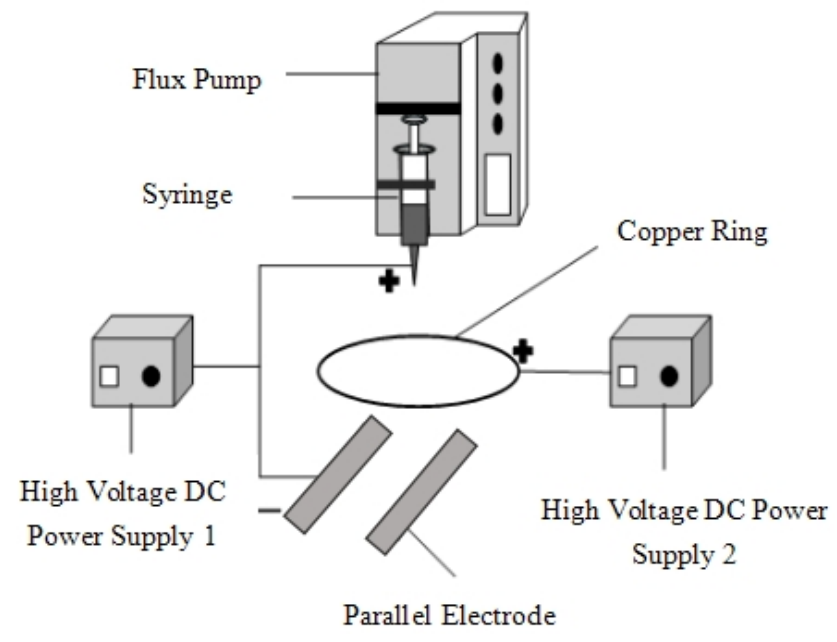

Fig. 1 The device of electrospinning

Electrospinning. A 8wt\% solution of PLA was spun with the instrument in Fig. 1 at $25+3{ }^{\circ} \mathrm{C}$ and $65 \%+5 \% \mathrm{RH}$, PLA solution was placed in $10 \mathrm{ml}$ vertically aligned disposable syringe having flat tip metallic needle which was controlled by syringe pump at a constant flow rate of $0.8 \mathrm{ml} / \mathrm{h}$. The needle was supplied a constant high voltage of $15 \mathrm{kV}$. The distance between the needle and the collector was $18 \mathrm{~cm}$. The copper ring with the diameter of $19 \mathrm{~cm}$ was subjected to a constant high voltage $5 \mathrm{kV}$, and the distance between it and parallel electrodes was $6 \mathrm{~cm}$. The electrodes were separated at a distance of $4 \mathrm{~cm}$.

\section{Results and Discussion}

The morphologies of electrospun fibers produced using the traditional electrospinning (ES) method and the MPEM were investigated respectively by scanning electron microscope (SEM), and SEM micrographs of the electrospun fibers with different electrospinning methods were illustrated in Fig. 2. 

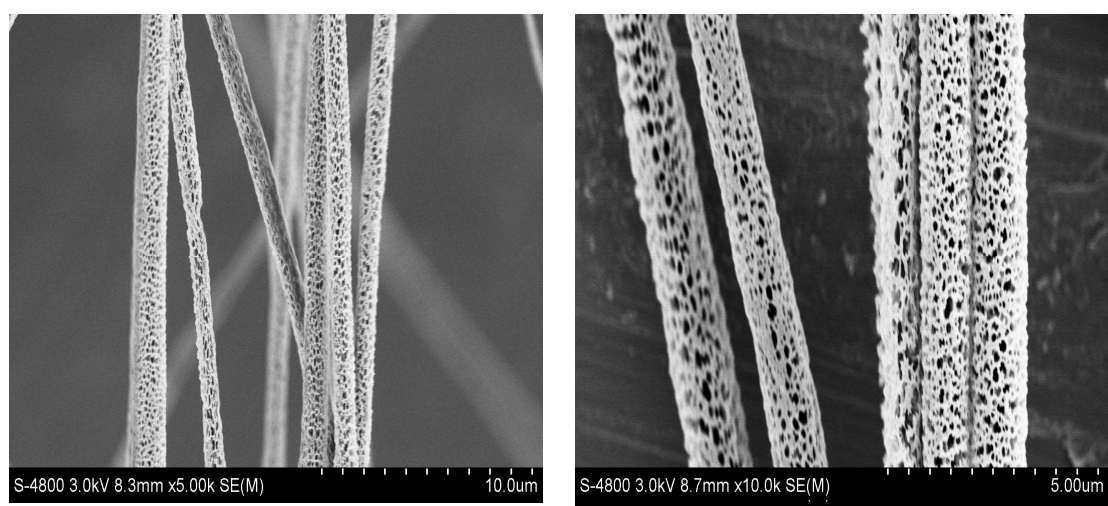

(a) SEM micrographs of the electrospun fibers using the ES
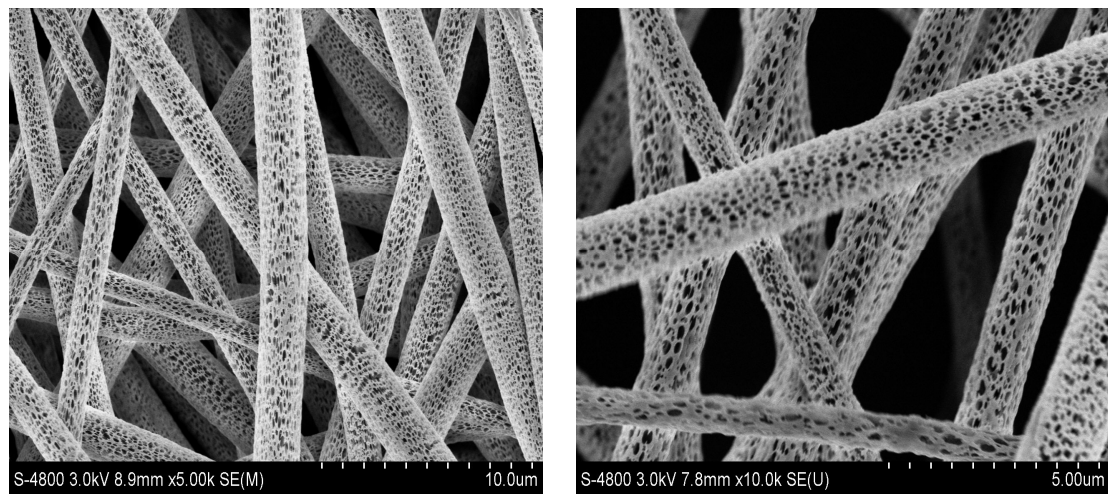

(b) SEM micrographs of the electrospun fibers using the MPEM

Fig. 2 SEM images of 8wt\% PLA solution system

It could be seen that aligned porous fibers could be produced by the MPEM, and the average diameters of the fibers was smaller than the ES. It was possible that the positively charged ring could decrease the energy loss of the charged jet in the electrospinning process. Therefore,more energy made the jet move faster in the electric field and the diameter became smaller [14]. Liu [15] had proved that adding a ring with positive before collector could reduce the diameter of fibers effectively.

\section{Conclusions}

It was proved that aligned porous fibers could be fabricated based on a ratio of 9:1 of the mixed solvent of CF and DMF by adding a copper ring and two metallic parallels. Pores could be observed obviously because of the different speed of volatilization of the two solvents. The extent of high-aligned array and the size and uniformity of pores should be explored by changing the parameters of electrospinning in the future.

\section{Acknowledgements}

The work is supported financially by PAPD (A Project Funded by the Priority Academic Program Development of Jiangsu Higher Education Institutions), Jiangsu Provincial Natural Science Foundation of China (Grant No. BK20131175), Natural Science Foundation of the Jiangsu Higher Education Institutions of China (Grant No. 14KJA130001), and Suzhou Science and Technology Project (Grant No. SYG201434).

\section{References}

[1] Q.J. Niu, L.W. Zeng, X.Y. Mu, J. Nie, G.P. Ma: Journal of Industrial and Engineering Chemistry. Vol. 34 (2016), p. 337 
[2] A. Haider, S. Haider, I.K. Kang: Arabian Journal of Chemistry. (2015), p. 1

[3] F.H. Anka, K.J. Balkus J: Industrial and Engineering Chemistry Research. Vol. 52 (2013), p. 3473

[4] D.Y. Yang, B. Lu, X.Y. Jiang: Advanced materials. Vol. 19 (2007), p. 3702

[5] J.M. Deilzed, J. Kleinmeyer, J.K. Hirvonmen, N.C. Beck Tan: Polymer. Vol. 42 (2001), p. 8163

[6] Z.D. Wu, D.H. Sun: Chinese Journal of Sensor and Actuators. Vol. 19 (2006), p. 1803

[7] D. Li, Y.L. Wang, Y.N. Xia : Nano. Lett. Vol. 3 (2003), p. 1167

[8] S. Zhong,W.E. Teo, X. Zhu, et al: J Biomed Mater Res Part A.Vol. 79A (2006), p. 456

[9] C.Y. Xu, R. Inai, M. Kotaki, S. Ramakrislma: Bio-materials. Vol. 25 (2004), p. 877

[10] J. Xu, J.H. Zhang, W.Q. Gao, H.W. Liang, H.Y. Wang, J.F. Li: Materials Letters.Vol. 63 (2009), p. 658

[11] Y. Li, Ch.T. Lim, M. Kotaki: Polymer.Vol. 55 (2014), p. 3970

[12] H.W. Zhang, Q.J. Niu, N. Wang, J. Nie, G.P. Ma: European Polymer Journal.Vol. 71 (2015),p. 440

[13] A. Kiziltas, B. Nazari, E.E. Kiziltas, D.J. Gardner,Y. Han, T.S. Rushing: Carbohydrate Polymers. Vol. 140 (2016), p. 393

[14] H.Y. Liu, L. Xu, N. Si: Therm. Sci. Vol. 18 (2014), p. 1451

[15] J.H. Zhao, H.Y. Liu, L. Xu: Materials and Design. Vol. 90 (2016) , p. 1 